\title{
An Exploration of the Tully-Fisher Relation for Extreme Late-Type Spiral Galaxies
}

\author{
L. D. Matthews \\ Department of Physics \& Astronomy, State University of New York at Stony Brook, Stony Brook, \\ NY 11794-3800 \\ Electronic mail: matthews@gremlin.ess.sunysb.edu \\ W. van Driel \\ Unité Scientifique Nançay, CNRS USR B704, Observatoire de Paris, 92195 Meudon, France \\ Electronic mail: vandriel@mesioq.obspm.fr \\ J. S. Gallagher, III \\ Department of Astronomy, University of Wisconsin-Madison, Madison, Wisconsin 53706 \\ Electronic mail: jsg@tiger.astro.wisc.edu
}

\begin{abstract}
In an earlier paper (Matthews et al. 1998) we presented new high-precision H I velocity width measurements for a sample of 30 extreme late-type spiral galaxies. Here we explore the adherence of those galaxies, as well as 17 additional extreme late-type spirals, to the $B$ - and $V$-band Tully-Fisher relations defined by a sample of local calibrators. In both bands we find the mean luminosity at a given linewidth for extreme late-type spirals to lie below that predicted by standard Tully-Fisher relations. While many of the extreme late-type spirals do follow the Tully-Fisher relation to within our observational uncertainties, most of these galaxies lie below the normal, linear Tully-Fisher relation, and some are underluminous by more than $2 \sigma$ (i.e. $>1.16$ magnitudes in $V$ ). This suggests a possible downward curvature of the Tully-Fisher relation for some of the smallest and faintest rotationally supported disk galaxies. This may be a consequence of the increasing prevalence of dark matter in these systems. We find the deviation from the Tully-Fisher relation to increase with decreasing luminosity and decreasing optical linear size in our sample, implying that the physically smallest and faintest spirals may be a structurally and kinematically distinct class of objects.
\end{abstract}

Subject headings: galaxies: spiral-galaxies: statistics-dark matter 


\section{Introduction}

The Tully-Fisher (TF) relation (Tully \& Fisher 1977) is an empirical correlation which predicts that the luminosity $L$ of a disk galaxy is proportional to its maximum rotational velocity $V_{\max }^{\alpha}$, where $\alpha$ has been observationally established to be $\sim 3-4$ (e.g., Aaronson et al. 1979). In spite of the frequent use of the TF relation as a distance indicator, the physical origin of this relationship is still relatively poorly understood, and it remains unclear whether all rotationally supported disk galaxies, including late-type spirals and irregulars, obey a single luminosity-linewidth correlation. Since the mean physical parameters which characterize disk galaxies, including total luminosity, gas fraction, and bulge size, change along the Hubble sequence, testing whether or not TF holds in different classes of disk galaxies can indirectly offer important information about galactic disk structure and evolution (e.g., Romanishin et al. 1982; Persic \& Salucci 1991; Karachentsev 1991; Zwaan et al. 1995; Rhee 1996; Meurer et al. 1996; Matthews et al. 1997; Bershady 1997) and can help to test recent semi-analytic models for disk galaxy formation (e.g., White \& Frenk 1991, Heyl et al. 1995).

In an earlier paper, Matthews et al. (1998; hereafter Paper I) presented new precise H I linewidth measurements for a sample of 30 southern, extreme late-type spiral galaxies】 with $V_{h} \leq 3000 \mathrm{~km} \mathrm{~s}^{-1}$. These objects are Sc-Sm spirals with moderate-to-low optical surface brightnesses and which fall at the low end for spiral galaxies in terms of properties such as $\mathrm{HI}$ mass, optical luminosity, and optical size. $B$ - and $V$-band CCD imaging and photometry of these galaxies was also recently published by Matthews \& Gallagher (1997; hereafter MG). Together these new datasets allow us to investigate the TF relation for extreme late-type spiral galaxies. Findings reported by MG, and in Paper I, suggest that some of the smallest spiral galaxies (i.e., the extreme late types) appear to be structurally and kinematically distinct from giant spirals (see also Matthews \& Gallagher 1998). Therefore it is of interest to assess whether or not they follow the same TF relation.

\section{The Luminosity-Linewidth Relation for Extreme Late-Type Spiral Galaxies}

Matthews et al. (1997) presented a preliminary analysis of the TF relation for 54 extreme late-type (ELT) spiral galaxies. Based on a comparison with a sample of high surface brightness galaxies from the Nearby Galaxies Catalog (Tully 1988; hereafter the TNGC), their finding was that there may be a curvature or an offset in the TF relation among some of the smallest and faintest spirals. Here we re-examine this trend more rigorously using the new, improved linewidths for the sample of galaxies presented in Paper I. We consider only those 27 galaxies with $i>30^{\circ}$. To increase our sample size, we also include 17 extreme late-type spirals with $i>35^{\circ}$ from the MG

\footnotetext{
${ }^{1}$ Matthews \& Gallagher (1997) define extreme late-type spirals as "the lowest luminosity, late-type disk galaxies which still exhibit regular optical structures and centralized light concentrations."
} 
photometric sample, for which H I linewidths are available in the literature (Fouqué et al. 1990; Gallagher et al. 1995). Hereafter we collectively refer to these 44 galaxies as the "combined ELT sample".

\subsection{The "Combined ELT Sample" and Corrections to Observed Quantities}

\subsubsection{Internal and External Extinction Corrections}

For the present analysis, we adopt the apparent $B$ and $V$ magnitudes of MG for the combined ELT sample galaxies. For consistency with other workers, we apply a correction to these magnitudes for Galactic extinction, $A_{B}$ (taken from Lauberts \& Valentijn 1989), and for internal extinction: $A_{B}^{i-0}=A_{B}^{i}-A_{B}^{0}$, where

$$
A_{B}^{i}=-2.5 \log \left[f\left(1+e^{-\tau \sec i}\right)+(1-2 f)\left(\frac{1-e^{-\tau \sec i}}{\tau \sec i}\right)\right],
$$

$A_{B}^{0}=0.27, \tau=0.55, f=0.25$, and $i$ is the galaxy inclination (Tully \& Fouqué 1985; hereafter, TFq). For $i>80^{\circ}, A_{B}^{i-0}=0.67$ is adopted (TFq), and we assume $A_{V}=A_{B} / 1.33$ (Allen 1963). We note that this approximate correction for $V$ formally applies only the limit of a foreground dust screen (the "normal extinction" case; see Calzetti et al. 1994). However, given the low optical depths considered here and the uncertain dust geometries in extreme late-type spirals, this approximation should be satisfactory (see also Han 1992). Nonetheless, we caution that our derived extinction corrections may be overestimates of the internal extinction for many moderate-to-low surface brightness galaxies (Rhee 1996; Pierce 1998; Sect. 2.4.2). If extinction is overestimated, then our derived luminosities will be too high.

\subsubsection{Distance Determinations}

We have computed absolute magnitudes for the combined ELT sample galaxies using distances derived via two methods: from a simple linear Hubble law and from galaxy group assignments, using the prescription of Tully et al. (1992) (see Sect. 2.4.5). To derive the linear Hubble flow distances, we used radial recessional velocities corrected for motion with respect to the Local Group according to Sandage \& Tammann (1981): $V_{c o r}=V_{h}+\Delta V$, where $\Delta V=-79 \cos \left(l_{I I}\right) \cos \left(b_{I I}\right)+296 \sin \left(l_{I I}\right) \cos \left(b_{I I}\right)-36 \sin \left(b_{I I}\right) \mathrm{km} \mathrm{s}^{-1}$. Here $V_{h}$ is the measured heliocentric recessional velocity, and $l_{I I}$ and $b_{I I}$ are the Galactic latitude and longitude, respectively. For consistency with Pierce \& Tully (1988), we adopt a Hubble constant of $85 \mathrm{~km} \mathrm{~s}^{-1} \mathrm{Mpc}^{-1}$. 


\subsubsection{Corrections to Observed Linewidths}

It is generally argued that in order to obtain a measure of the maximum rotational velocity of a disk from its measured global H I profile width, $W_{20}$, some correction to the observed line width should be made for the effects of broadening due to turbulent (i.e. non-rotational) motions (e.g., Roberts 1978; Bottinelli et al. 1983; TFq). A commonly used formulation of this correction, suggested by $\mathrm{TFq}$, assumes that a linear summation of rotational and random motions adequately describes the observed profile widths of giant galaxies, while for dwarfs (i.e., slowly rotating galaxies with Gaussian-like line profiles), a sum in quadrature of the the random and rotational terms is appropriate. However, the TFq method has serious shortcomings (Rhee 1996), and it is not necessarily appropriate for the types of objects considered here.

Our combined ELT sample consists of galaxies that appear disk-like and regular in optical images (MG). And while many of the systems have fairly narrow $\mathrm{H}$ I line profiles, all exhibit significant rotational broadening and most are double-peaked (Paper I). Moreover, nearly "Gaussian" profiles can still occur for small, non-face-on galaxies supported predominantly by rotation (e.g., Skillman 1996). Thus linewidth corrections optimized for "dwarfs" are unlikely to be appropriate for our sample galaxies. For this reason, we adopt the linear turbulence correction formula suggested by Bottinelli et al. (1983):

$$
W_{20, i, c}=\left[W_{20, o b s}-W_{t, 20}\right] \times(\sin i)^{-1}
$$

where $W_{20, o b s}$ is the observed profile with at $20 \%$ peak maximum, corrected for instrumental broadening, $W_{t, 20}$ is the correction term for turbulence, and the factor of $\sin i$ corrects for disk inclination. For the combined ELT sample, we use the optically derived inclinations from MG.

A second matter of debate for computing linewidth corrections is the optimum value of $W_{t, 20}$. $\mathrm{TFq}$ assume a typical velocity dispersion in the gas of a galaxy is $\sigma_{z}=10 \mathrm{~km} \mathrm{~s}^{-1}$, leading to a derived turbulent motion term $W_{t, 20}=38 \mathrm{~km} \mathrm{~s}^{-1}$ (TFq). However, $\sigma_{z}=10 \mathrm{~km} \mathrm{~s}^{-1}$ is at the high end for observed $\mathrm{H}$ I velocity dispersions in the outer disks of either dwarf or spiral galaxies, where typically $\sigma_{z} \sim 5$-10 $\mathrm{km} \mathrm{s}^{-1}$ (e.g., Dickey et al. 1990; Kamphuis \& Sancisi 1993; Rownd et al. 1994; Skillman 1996; Young \& Lo 1996; Côté et al. 1997; Pisano et al. 1998).

From a comparison between global $\mathrm{H}$ I linewidths and maximum rotational velocities derived from rotation curves, Rhee (1996) statistically derived a smaller value for the turbulent motion correction term of $W_{t, 20}=20 \mathrm{~km} \mathrm{~s}^{-1}$ (corresponding to $\sigma_{z} \sim 5.5 \mathrm{~km} \mathrm{~s}^{-1}$ in the case of a nearly Gaussian velocity dispersion). Although the uncertainty in this term for any individual galaxy can be large, Rhee (1996) has shown that $W_{t, 20}=20 \mathrm{~km} \mathrm{~s}^{-1}$ should be the optimum value for recovering $V_{\max }$ for a statistical sample of galaxies; we therefore adopt this number in the present work. 


\subsection{The Comparison Sample}

As a TF comparison sample, we have chosen the standard local calibrator sample of Pierce \& Tully (1992; hereafter PT). We used the absolute $B$ magnitudes quoted in their paper (corrected for internal and external extinction as outlined above), and have derived absolute $V$ magnitudes using the internal and external extinction-corrected $B-V$ colors from the RC3 (de Vaucouleurs et al. 1991). We derived $W_{20, i, c}$ values for the PT sample from the raw $W_{20}$ values and inclinations quoted by PT. All corrections we apply to the PT sample are necessary for consistency with the corrections applied to the combined ELT sample.

Cepheid distances are available for only 6 galaxies in the PT sample, and PT caution against calibrating the TF relation using their full sample. However, we find the relation we derive from the full sample of PT galaxies to be statistically indistinguishable from that derived from the Cepheid galaxies alone, apart from its larger scatter. Therefore for the purpose of our analysis, we consider the full PT sample, as it yields a better estimate of the intrinsic scatter one might expect in a sample of galaxies for which independent distance determinations are not available.

\subsection{The Luminosity-Linewidth Distribution for the Combined ELT Sample}

Fig. $1(a)$ and $(b)$ show plots of the $B$ - and $V$-band absolute magnitude versus the logarithm of the corrected H I linewidth for the 27 extreme late-type spirals from Paper I (triangles), the 17 extreme late-type spirals from MG (squares), and the PT comparison sample (asterisks). The absolute magnitudes for our combined ELT sample galaxies are based on distances derived from a simple linear Hubble flow model (Sect. 2.1.2); other distance determination methods are considered in Sect. 2.4.5.

We performed a linear fit to the PT points using the ordinary least squares bisector method, which minimizes the residuals in both $x$ and $y$. For small data sets, this is the preferred method for finding the intrinsic relationship between two variables in the case where errors for the individual data points are not explicitly known (Babu \& Feigelson 1996). The results of the fits are: $M_{B, i, b}=-\left[(6.12 \pm 0.37) \times\left(\log \left(W_{20, i, c}\right)\right)\right]-(3.82 \pm 0.88)$ and $M_{V, i, b}=-\left[(6.53 \pm 0.25) \times\left(\log \left(W_{20, i, c}\right)\right)\right]-(3.56 \pm 0.58)$. These fits are plotted as solid lines on Fig. 1; the dotted lines indicate the $2 \sigma$ scatter. Our resulting $B$-band fit differs very slightly from that of PT, as we used a different method for determining turbulent motion corrections. PT did not present a $V$-band TF relation for their data, but for normal galaxy colors, our $V$-band fit is consistent with the expected offsets in both slope and y-intercept from the PT $B$-band and $R$-band calibrations.

Inspection of Fig. 1 reveals that while many of the galaxies from the combined ELT sample scatter about the TF relations defined by the PT galaxies, there is a mean offset of the ELTs toward fainter magnitudes in both $B$ and $V$. In fact, a number of the combined ELT sample 
galaxies scatter more than $2 \sigma$ below the fits to the PT galaxies. Because our combined ELT sample covers a relatively narrow range in $\log \left(W_{20, i, c}\right)$, a linear least squares fit to these data does not have a well-constrained slope. For this reason, we do not attempt to analytically fit our combined ELT sample, but instead consider the distribution of the combined ELT sample points relative to the TF relation defined by the local calibrator samples.

\subsection{Does the Combined ELT Sample Obey the TF Relation to within Observational Uncertainties?}

The mean offsets of the combined ELT sample galaxies from the standard $B$ - and $V$-band TF relations are 0.64 and 0.82 magnitudes, respectively. The reduced chi-squared values resulting from forcing the fits to the PT local calibrators to the combined ELT sample are $\chi_{\nu, B}^{2}=1.40$ (for $V$ ) and $\chi_{\nu, V}^{2}=1.72$ (for $B$ ). If we assume the local calibrator fits should adequately characterize the combined ELT sample, there is only a $5 \%$ probability of obtaining $\chi_{\nu, B}^{2}$ this large or larger from a randomly selected set of points, and a $0.1 \%$ probability of obtaining or exceeding this value of $\chi_{\nu, V}^{2}$. Thus it is highly probable that the standard TF relation does not represent the true underlying distribution for the combined ELT sample data points in Fig 1. Before we can establish whether this observed offset from TF is real, we must rule out observational errors and biases which may account for part or all of this effect. We discuss various possibilities in turn.

\subsubsection{Photometric Uncertainties}

MG present a comparison of their measured $B$-band isophotal magnitudes with those in the ESO Catalogue (Lauberts \& Valentijn 1989) and found a mean offset of $\sim+0.2$ magnitudes, consistent with offsets from the ESO magnitude system found by other workers (e.g., Rönnback \& Bergvall 1994; Vader \& Chaboyer 1994; Vennik et al. 1996). Uncertainties in individual magnitude measurements are typically $<0.03$ magnitudes. Therefore photometric calibration uncertainties are unlikely to contribute a significant offset from TF for the combined ELT sample.

Still, one concern is that for the faintest galaxies in the combined ELT sample, the measured magnitudes may not adequately represent the true total magnitudes, since for galaxies of very low

central surface brightness, a significant percentage of the total flux lies in the faint, outer portions of the disk and may be unmeasurable on CCD images (e.g., McGaugh \& Bothun 1994). We estimate that such corrections would be less than $\sim 0.1$ magnitude for our galaxies (see de Blok et al. 1995; Tully et al. 1996), yielding a total uncertainty in our absolute magnitudes of $\sim 0.33$ magnitudes. 


\subsubsection{The Use of $B$ and $V$ Photometry and the Effects of Internal Extinction}

In theory, it is preferable to perform TF analyses using near-infrared (NIR) rather than optical magnitudes in order to minimize magnitude uncertainties due to internal and external extinction and to reduce sensitivity to the effects of recent star formation. However, for faint galaxies, the high and variable sky background in the NIR and the large aperture corrections which are necessary at these wavelengths place serious limits on achievable photometric accuracy. The small field sizes of most currently available NIR detectors can also lead to large flatfielding errors (generally the dominant source of error in the photometry of faint, large angular size objects). Increasing sky and field star brightnesses as well as flatfielding difficulties at $R$ and $I$ also create problems with using these wavebands for TF studies of faint galaxies. For these reasons, $B$-band and $V$-band make reasonable choices for exploring the TF relation for extreme late-type spirals, although investigations at other wavelengths will naturally be desirable as photometry becomes available.

The need to move to longer wavelengths for TF investigations is also lessened for extreme late-types spirals because these galaxies appear to have fairly low internal extinction (e.g., McGaugh 1994; Rönnback \& Bergvall 1995; Bergvall \& Rönnback 1995; de Blok et al. 1995; Tully et al. 1998). One way of seeing that internal extinction is likely to be low for many of our combined ELT sample objects is to compare their extinction-corrected $B-V$ colors with those of higher surface brightness galaxies. Fig. 2 shows a plot of $B-V$ color, derived from our internal and external extinction-corrected magnitudes, versus $\log \left(W_{20, i, c}\right)$ for our combined ELT sample (squares and triangles). As a comparison, we show the PT local calibrator galaxies (asterisks) and a sample of galaxies from the TNGC (crosses). The TNGC sample consists of all galaxies in the TNGC that lie within the same redshift range $\left(V_{h}<3000 \mathrm{~km} \mathrm{~s}^{-1}\right)$ and same angular volume $\left(3^{h} \leq \alpha \leq 14.5^{h},-18^{\circ} \leq \delta \leq-38^{\circ}\right)$ as our combined ELT sample. We see that many extreme late-type spirals appear to be among the bluest galaxies at a given linewidth, although they span a range in color. Since bluer galaxies generally have less dust and younger stellar population mixes, both of these effects might be expected to cause galaxies to lie above the standard TF relationship -i.e. to be too bright for their rotation speeds. However, Fig. 3 shows that there is no apparent correlation between extinction-corrected $B-V$ color and deviation from the TF relation, $(\Delta T F)_{V}$, in our sample.

Further inspection shows that the extinction corrections applied to the combined ELT sample have in some cases lead to unreasonably blue $B-V$ colors $\left[(B-V)_{i, b}<0.1\right]$, implying that the internal extinctions estimated using the method of $\mathrm{TFq}$ are overestimates for at least some extreme late-type spirals. Thus the TFq extinction corrections appear to have artificially "brightened" some of our combined ELT sample galaxies, and worked to decrease any possible intrinsic offsets from TF. The inappropriateness of the TFq corrections for very late-type galaxies has also been cited by Rhee (1996) and Pierce (1998). In addition, these workers note that the TFq corrections may be underestimates for some very bright galaxies, hence further masking possible intrinsic TF offsets between different galaxy samples or possible curvature in the TF relation. 


\subsubsection{Uncertainties in $W_{20}$ Measurements}

For the 27 galaxies from Paper I, uncertainties in $W_{20}$ range from 1-23 $\mathrm{km} \mathrm{s}^{-1}$. Similar errors are quoted for the $W_{20}$ values from the literature for the remaining 17 galaxies in the combined ELT sample, as well as for the PT calibrators. This should lead to maximum errors in predicted TF magnitudes of no more than a few tenths of a magnitude for individual galaxies.

\subsubsection{Inclination Errors}

Even when linewidths are measured very accurately, uncertainties are introduced in correcting these velocity widths for inclination. Most of our optically-derived inclinations should be good to $\pm 5^{\circ}$ (MG), but for some galaxies, errors could be larger due to the significant optical asymmetries, warps, or irregular outer isophotes which are common in extreme late-type spirals. Such effects may increase the scatter in TF by a few tenths of a magnitude (Franx \& de Zeeuw 1992). However, we argue that there is unlikely to be a systematic trend toward the overestimation of rotational velocities from inclination effects. Optical inclinations are generally derived using the axial ratio of the observed isophotes, assuming that the intrinsic face-on galaxy isophotes would be circular. However, if a galaxy has intrinsically non-circular isophotes (as is often the case for very late-type spirals), even if it is observed face-on, the inclination of the galaxy will be measured to be non-zero; this effect should become less pronounced as the galaxy is turned edge-on. Therefore in most cases, inclination errors should be in the sense of the inclinations being overestimated, and the galaxy's corrected rotational velocity being underestimated.

In Fig. 4 we show a plot of $(\Delta T F)_{V}$ versus inclination. Here we see only a very slight correlation, which is small compared with the scatter, and we find TF deviators among the highest $i$ galaxies (i.e., the galaxies with the smallest inclination corrections to their total linewidths). We note that one of our largest TF deviators (ESO 504-017) also has one of the smallest inclinations $(i=30.8)$ in our sample. However, we retain this galaxy in our analysis, since even for $i \approx 60^{\circ}$ it would still fall more than $2 \sigma$ below the standard $V$-band TF relation.

\subsubsection{Distance Uncertainties}

A major drawback of analyzing TF in a nearby galaxy sample is that deviations from a simple linear Hubble flow may occur due to local peculiar velocities, leading to errors in distance determinations. This is likely to be the largest source of uncertainty in our present analysis.

Unfortunately, current characterizations of the local peculiar velocity field are still incomplete and uncertain on small scales (e.g., Shaya et al. 1992 and references therein). Nonetheless, we need to examine the possibility that some or all of the apparent offset of extreme late-type galaxies from the standard TF relation is due to errors in distances caused by peculiar velocity motions. 
One recent model of nearby galaxy flows was presented by Shaya et al. (1992). In a companion paper, Tully et al. (1992) used this model to derive distances to many of the galaxy groups defined in the TNGC. As suggested by Tully et al. (1992), distances to other Local Supercluster galaxies may be determined by assigning the galaxy in question to a TNGC group. We therefore utilize this approach to estimate peculiar velocity-corrected distances for our combined ELT sample galaxies.

Using Tully \& Fisher (1987) and the TNGC, we were able to make group assignments to 39 of our combined ELT sample galaxies (Table 1). Tully et al. (1992) provide distances for 32 of these groups. In addition, 5 of our galaxies lie within a $6^{\circ}$ projected radius from the center of the Fornax cluster. For these objects, we adopt as the distance the current best-estimate for the distance to the Fornax cluster of $18.3 \mathrm{Mpc}$ (Freedman et al. 1997; Silbermann et al. 1998). Bureau et al. (1996) have shown that normal galaxies within $6^{\circ}$ of the Fornax center follow the same TF relation as other nearby clusters, so a priori we do not expect to see unusual deviations among these objects.

Adopting the group distances given in Table 1, we present a revised $V$-band TF relation in Fig. 5. Here we see that while there is some reduction in scatter, there is still a mean offset of 0.69 magnitudes for the combined ELT sample. Although the ELT sample plotted here is smaller than in Fig. 1, we still find 9 galaxies that are underluminous by $>2 \sigma$ compared with the prediction of the TF relation. Therefore, current peculiar velocity models cannot reconcile the apparent offset of the bulk of extreme late-type spirals from the standard TF relation. Given the uncertainty in our present knowledge of the local peculiar velocity field, we of course cannot rule out that in a few individual cases, distance errors may be responsible for the observed offsets. Overall, we estimate typical uncertainties in absolute magnitude due to distance errors of $\sim \pm 0.45$ magnitudes, which is the mean difference in the absolute magnitudes determined by the linear Hubble flow method versus the group assignment method.

Another way of assessing the effect of distance errors on our TF comparison is to examine the relationship between absolute magnitude and linear size $\left(A_{26}\right)$ for our sample. Erroneous distances that cause errors in the derivation of absolute magnitudes will also cause errors in the derived linear sizes of the galaxies. In Fig. $6(a)$ we plot absolute $V$ magnitude for our combined ELT sample galaxies versus the logarithm of the linear diameter, $A_{26}$, in kiloparsecs. Both of these quantities are derived assuming linear Hubble flow distances. In Fig. $6(b)$ we plot the same quantities, but this time we have assigned to each galaxy a revised distance assuming it adheres to the standard TF relation. It is clear in Fig. $6(b)$ that forcing our galaxies onto the TF relation results in a very unrealistic distribution of linear sizes versus absolute magnitudes. Galaxies with $M_{V, i, b} \sim-18$ appear to span a factor of 12 is diameter, and the physically smallest galaxies in our sample would have to span a range of almost 6 magnitudes in brightness. Again this suggests that our observed offset from the TF relation cannot be fully accounted for by distance errors. 


\subsection{Discussion}

We conclude that systematic errors are unlikely to be the source of the global offset of the combined ELT sample from the standard TF relation. Summing in quadrature our expected sources of error, including distance uncertainties ( \pm 0.45 magnitudes), photometric uncertainties ( \pm 0.33 magnitudes), $W_{20}$ measurement uncertainties ( \pm 0.25 magnitudes), internal extinction uncertainties ( \pm 0.2 magnitudes), and inclination effects ( \pm 0.3 magnitudes), we estimate a typical error budget for the comparison of any individual galaxy with $\mathrm{TF}$ of $\sim \pm 0.71$ magnitudes in the $B$ band. In the $V$ band, we should also take into account possible magnitude errors for the PT galaxies due to aperture corrections of $\sim \pm 0.15$ magnitudes (de Vaucouleurs et al. 1976), yielding a total uncertainty of \pm 0.72 magnitudes for each individual galaxy. While we cannot rule out that in a few cases, distance errors, combined with observational uncertainties may produce spurious deviations from $\mathrm{TF}$, the typical errors that we estimate are insufficient to account for the large number of galaxies we find that deviate by more than $2 \sigma$ from the standard TF relation. In addition, we emphasize that errors in inclinations and internal extinction estimates may have actually decreased any intrinsic offset of some of our combined ELT sample objects from TF.

The type of observed offset from TF we see among our extreme late-type spirals is consistent with past indications of a curvature or offset at the faint end of TF for other low surface brightness (LSB) and/or low-luminosity galaxies in the sense of these galaxies being underluminous for a given value of $W_{20}$ (e.g., Romanishin et al. 1982; Carignan \& Beaulieu 1989; Persic \& Salucci 1991; Salpeter \& Hoffman 1996; Meurer et al. 1996; Freeman 1997; Salucci \& Persic 1997; Walsh 1998).

MG have emphasized that there exists a significant range in the physical properties of extreme late-type spirals (see also Paper I). Consistent with this assertion, even if we assume that the majority of the combined ELT sample galaxies are in accordance with the standard, linear TF relation to within observational uncertainties, this still leaves at least 9 galaxies in our combined ELT sample that lie more than $2 \sigma$ below the standard $V$-band TF calibration, even after corrections to distances for peculiar velocity motions (Fig. 5). Therefore, among the extreme late-type spirals there appear to exist examples of rotationally-supported disk galaxies that are underluminous for their observed rotational velocities.

Because very late-type galaxies can frequently have dynamics dominated more by the gaseous than the stellar components (e.g., Jobin \& Carignan 1990; Côté et al. 1991; Meurer et al. 1996), Milgrom \& Braun (1988) have suggested adding to the optical luminosity a correction for the H I "luminosity" or a "baryonic correction" in order to preserve the TF relation for certain galaxies.

Using the PEGASE stellar evolutionary code of Fioc \& Rocca-Volmerange (1997), we estimate a typical $(\mathcal{M} / L)_{\star}$ ratio for extreme late-type spirals of $\sim 1$ (see also Bica et al. 1988). Assuming a helium fraction of $\mathrm{Y}=0.25$, and negligible molecular gas contents, we apply to all of our combined ELT sample galaxies, as well as to the PT calibrator galaxies, a baryonic correction of the form:

$$
L_{\text {bary }}=L_{V}+1.33 \mathcal{M}_{\mathrm{HI}} \text {. }
$$


We see in Fig. 7 that applying the baryonic correction can reconcile some but not most of the combined ELT sample galaxies with the TF relation defined by the PT local calibrators. We still see a global offset of the combined ELT sample of 0.63 magnitudes relative to the local calibrators, since the baryonic correction also shifts to brighter magnitudes the late-type galaxies that anchor the faint-end of the local calibrator sample. In addition, the baryonic correction does not significantly reduce the scatter in the TF relation for the combined ELT sample galaxies, and greatly increases the dispersion of the PT galaxies about the best-fit power law.

In Fig. 8 and 9 we see that there exist only weak correlations between both $\frac{\mathcal{M}_{H I}}{L_{V}}$ and mean disk surface brightness, $\bar{\mu}_{V}$, and the deviation from the TF relation, $\Delta(T F)_{V}$ in the combined ELT sample. Together these results suggest that while to some degree, our TF deviators appear to be galaxies that have been inefficient at converting their raw material into stars (cf. Hunter \& Gallagher 1986), this cannot fully account for the deviation from the TF relation for most of our sample objects (see also Meurer et al. 1996). These findings hint that the combined gas and stars in the inner galaxy is not what primarily determines $V_{\max }$ of the rotation curves for some of our extreme late-type spirals - i.e., these galaxies may be dark matter-dominated even within the optical galaxy. Similar results have also emerged from recent rotation curve studies of these objects (e.g., Matthews \& Gallagher 1998). Such effects are expected, for example, if the degree of luminous-to-dark matter coupling decreases among low-mass galaxies (Salucci \& Persic 1997). We discuss this possibility further in the next section.

\subsubsection{The Possible Role of Dark Matter}

Using the parameters given by Carignan \& Beaulieu (1989) and Meurer et al. $(1994,1996)$, we have overplotted on Fig. 1 (a) two additional small, faint disk galaxies: NGC 2915 and DDO 154. It is clear that both of these galaxies deviate significantly from the standard $B$-band TF relation. Meurer et al. (1996) has discussed the likelihood that the deviations of NGC 2915 and DDO 154 from $\mathrm{TF}$ are a consequence of the high dark matter domination of these galaxies. Could this also be the case for our TF deviators?

In Fig. 10 we plot $\Delta(T F)_{V}$ versus $V$-band optical linear size $\left(A_{26}\right)$ in kpc for our combined ELT sample. Here we see an apparent tendency for the smallest galaxies to be the greatest deviators from the TF relation. Since for a gravitationally-supported disk, if all the mass is visible, then $V_{\text {rot }}^{2} r \propto L$, where $V_{\text {rot }}$ is the rotational velocity, $r$ is galactocentric distance, and $L$ is the luminosity. Therefore smaller galaxies with a given $L$ should rotate faster. The observed adherence of most disk galaxies to a universal TF relation implies that a given amount of optically luminous matter is always radially scaled to produce a set, corresponding rotational velocity-i.e. that the product of the galaxy surface density $\bar{\Sigma}$ and the mass-to-light ratio $\mathcal{M} / L$ is a constant (e.g., Zwaan et al. 1995). However, this trend may break down among some of the smallest spirals (e.g., Kormendy 1990; Salucci \& Persic 1997). 
Our present findings suggest that at a given rotational velocity, the smallest galaxies are physically distinct from the largest ones, and that total matter distributions (or $\bar{\Sigma}$ ) are not always simply radially scaled as to preserve a linear TF relation. A natural interpretation of this is that the most severely TF-deviating galaxies in our sample are likely to be galaxies where the luminous matter distribution ( $\mathrm{H}$ I + stars) contributes the least to establishing the total rotational velocity of the galaxy, and where the luminous matter distributions are compact relative to the extent of the dark matter halo. Such phenomena are predicted by dynamical models for low-luminosity disks (Levine \& Sparke 1998), galaxy scaling laws (Kormendy 1990; see also Freeman 1997), and for the models of dark and luminous matter coupling in galaxies proposed by Salucci \& Persic (1997). Salucci \& Persic (1997) have also shown that their model predicts a downturn in the TF relation among low-luminosity, moderate-to-low surface brightness galaxies that is consistent with that observed in the extreme late-type spiral sample of Matthews et al. (1997).

Further indirect evidence that dark matter may play a role in the deviation of some of our sample galaxies from the standard TF relation comes from Fig. 11, where we plot absolute $V$ magnitude for our combined ELT sample galaxies versus $\Delta(T F)_{V}$. Here we see some indication that deviation from TF appears to increase with decreasing luminosity in our sample. This hints that the trend we are observing is indicative of a curvature rather than a global offset at the faint end of the TF relation (see also Salucci \& Persic 1997). This type of offset is consistent with the predictions of galaxy scaling laws, which show that the density of dark matter halos increases with decreasing galaxy luminosity, resulting in increasingly dark matter-dominated inner disks in fainter spirals (Kormendy 1990). Previous results of aperture synthesis studies have indicated that the increasing importance of dark matter starts to become evident at $M_{V} \approx-17.5$ (Skillman et al. 1987; Lake et al. 1990; Meurer et al. 1996), approximately the same point at which we see our sample galaxies beginning to fall consistently below the TF relation.

The true form of the TF relationship has important implications for testing the robustness of galaxy formation models. For example, Dalcanton et al. (1997) predict only a slight curvature in the TF relationship for their models of disk galaxies. They, however, find good agreement with observations in that they see only a very weak dependence of the TF relationship on disk surface brightness (cf., Fig. 9). Recent semi-analytic models by Mo et al. (1998), in which the authors seek to present a consistent picture of galaxy formation within a given cosmology, also show only a very slight curvature down from a linear TF relationship at low masses. However, a wider range of downward curvatures is seen, for example, in some of the models of Somerville \& Primack (1998). While detailed comparisons between global characteristics of low mass disk galaxies and predictions of formation models are beyond the scope of this paper, this issue clearly warrants future attention. 


\subsubsection{Comparison with Other Recent Observational Studies}

The results we present here for the TF relation of extreme late-type spirals may seem at first glance contrary to the conclusions reached in two recent investigations of the TF relation for LSB spirals: Sprayberry et al. (1995) and Zwaan et al. (1995). These authors find that their LSB spiral samples follow the same TF relation as high surface brightness spirals (see also de Blok et al. 1996). We argue that the difference is due primarily to the examination of a somewhat different class of galaxies than the present study: the predominantly "large" LSB galaxies versus the extreme late-type spirals.

The LSB galaxy samples studied by Zwaan et al. (1995), Sprayberry et al. (1995) and de Blok et al. (1996) are mainly composed of larger scale-length spirals with typical luminosities and H I masses larger than in our combined ELT sample. For example, in the de Blok et al. (1996) sample, the mean $\log \left(\mathcal{M}_{\mathrm{HI}}\right)$ is $9.23 \pm 0.27 \mathcal{M}_{\odot}$ and the mean absolute $B$ magnitude is $\bar{M}_{B}=-17.53 \pm 0.88$ (for

$\left.H_{0}=75 \mathrm{~km} \mathrm{~s}^{-1} \mathrm{Mpc}^{-1}\right)$, compared with a mean $\log \left(\mathcal{M}_{\mathrm{HI}}\right)$ of $8.78 \pm 0.50 \mathcal{M}_{\odot}$ and $\bar{M}_{B}=-16.56 \pm 1.14$ in the combined ELT sample. In the Sprayberry et al. (1995) sample, $\bar{W}_{20}=286 \pm 84 \mathrm{~km} \mathrm{~s}^{-1}$ (before inclination correction) while $\bar{W}_{20} / \sin i=146 \pm 52 \mathrm{~km} \mathrm{~s}^{-1}$ for our combined ELT sample. Because many of our combined ELT sample galaxies lie outside of the $M_{B}, W_{20}, \mathcal{M}_{\mathrm{HI}}$, and $A_{26}$ domains that typify the samples of Sprayberry et al. (1995), Zwaan et al. (1995), and de Blok et al. (1996), and since in many cases we find the most significant departures from TF for the smallest and faintest disks (see also Salpeter \& Hoffman 1996), this may explain why these workers did not find similar evidence for deviations from the TF relation in their samples.

\section{Summary and Conclusions}

Using new and previously published H I data, together with the photometry of Matthews \& Gallagher (1997; MG), we have explored the $B$ and $V$-band Tully-Fisher (TF) relation for 44 extreme late-type spiral galaxies. Relative to the TF relation defined by the local calibrator sample of Pierce \& Tully (1992), we find offsets of 0.64 and 0.82 magnitudes in $B$ and $V$, respectively, for our sample of extreme late-type spirals. For 37 of our sample galaxies, following Tully et al. (1992), we have derived revised distances based on assignments to galaxy groups whose distances have been previously determined using models for local peculiar velocity flows. The extreme late-type spirals with corrected distances still show a mean offset from TF of 0.69 magnitudes in $V$. Thus within the limits of present models, local peculiar motions cannot account for the effects we observe. Adopting distances to our sample galaxies by assuming they do lie on TF results in an unrealistic distribution of absolute magnitude versus linear diameter. This is further evidence that distance errors are not the source of the observed departure of many extreme late-type spirals from TF. Therefore, our data show evidence for the existence of populations of rotationally supported disk galaxies which lie below the normal, linear TF relation-i.e., these galaxies appear to be optically under-luminous for their rotation speeds. 
While offsets of individual galaxies from the TF relation of up to \pm 0.72 magnitudes may be explicable as artifacts of observational uncertainties, or individual distance determination errors, these effects cannot account for the observed global offset of our sample. Even after correcting distances for local peculiar motions, we still find 9 extreme late-type spirals that deviate by more than $2 \sigma$ (i.e. $>1.16$ magnitudes) from the $V$-band $\mathrm{TF}$ relation defined by the local calibrator sample.

We find that deviation from the TF relation, $\Delta(T F)$, appears to increase with decreasing linear optical size and decreasing luminosity in our sample, which suggests that the greatest TF deviators are galaxies where the luminous matter contents are small and compact relative to the size and extent of their dark matter halos and that there may be curvature at the faint end of the TF relation. We see only very weak correlations between $\Delta(T F)$ and inclination or mean disk surface brightness, and no obvious correlation between $\Delta(T F)$ and $B-V$ color. Including the $\mathrm{H}$ I flux as part of the total luminosity cannot reconcile most of these galaxies with the TF relation, and $\Delta(T F)$ does not correlate strongly with increasing $\frac{\mathcal{M}_{H I}}{L_{V}}$. These trends suggest that the TF deviators are not simply galaxies that have retained large gas reservoirs due to slow evolution, and hints that the TF deviators may be galaxies where the sum of the visible gas and stars contributes little to the determination of $V_{\max }$ of the rotation curve - i.e. they may be dark matter dominated even within the optical galaxy. Observations of these low mass disk galaxies may therefore provide useful constraints on galaxy formation models.

This work made use of data obtained at the Nançay Radio Observatory. The Nançay Observatory is the department Unité Scientifique Nançay of the Observatoire de Paris and is associated with the French Centre National de Recherche Scientifique (CNRS) as the Unité de Service et de Recherche (USR), No. B704. The Observatory also gratefully acknowledges the financial support of the Région Centre in France. We acknowledge useful discussions with M. J. Pierce and A. Yahil on aspects of this work and thank D. M. Peterson for a critical reading of an earlier version of this manuscript. JSG's work in this area benefitted from his participation in a workshop at the Aspen Center for Physics. LDM is supported by a graduate internship with the Wide Field and Planetary Camera 2 Investigation Definition Team, which is supported at the Jet Propulsion Laboratory (JPL) via the National Aeronautics and Space Administration (NASA) under contract No. NAS7-1260. This research made use of the NASA/IPAC Extragalactic Database (NED), operated by JPL under contract with NASA. 
Table 1.

\begin{tabular}{|c|c|c|}
\hline Galaxy & Group & $\mathrm{D}(\mathrm{Mpc})$ \\
\hline \multicolumn{3}{|c|}{ Paper I Galaxies } \\
\hline ESO 547-020 & $51-0+4$ & 22 \\
\hline ESO 418-008 & Fornax & 18.3 \\
\hline ESO 418-009 & Fornax & 18.3 \\
\hline ESO 482-005 & $51-4$ & 20 \\
\hline ESO 358-015 & Fornax & 18.3 \\
\hline ESO 358-020 & Fornax & 18.3 \\
\hline ESO 548-050 & $51-4$ & 20 \\
\hline ESO 549-002 & $53-0^{*}$ & $\cdots$ \\
\hline ESO 358-060 & Fornax & 18.3 \\
\hline ESO 359-016 & $51-0+18$ & 18 \\
\hline ESO 359-029 & $53-13$ & 17 \\
\hline ESO 359-031 & $53-13$ & 17 \\
\hline ESO 422-005 & $51-10$ & 18 \\
\hline ESO 305-009 & $53-10$ & 12 \\
\hline ESO 487-019 & $34-2$ & 20 \\
\hline ESO 488-049 & $34+1^{*}$ & $\cdots$ \\
\hline ESO 425-008 & $34-1$ & 21 \\
\hline AM0605-341 & $53-14$ & 9 \\
\hline ESO 431-015 & $\cdots$ & $\cdots$ \\
\hline ESO 497-007 & $31-0+12$ & 30 \\
\hline ESO 500-032 & $31-0+2$ & 30 \\
\hline ESO 502-016 & $22-0^{*}$ & $\cdots$ \\
\hline ESO 438-005 & $22-6$ & 14 \\
\hline ESO 504-017 & $22-4$ & 17 \\
\hline ESO 505-013 & $22-1$ & 17 \\
\hline ESO 380-025 & $23-5$ & 24 \\
\hline ESO 443-079 & $11-22$ & 16 \\
\hline ESO 443-080 & $11-22$ & 16 \\
\hline ESO 444-033 & $11-32$ & 20 \\
\hline ESO 446-053 & $16-0$ & 10 \\
\hline \multicolumn{3}{|c|}{ MG Galaxies } \\
\hline SCG0448-39 & $\ldots$ & $\ldots$ \\
\hline ESO 552-066 & $34-3$ & 22 \\
\hline NGC 2131 & $34+1^{*}$ & $\ldots$ \\
\hline ESO 377-017 & $31-10$ & 27 \\
\hline ESO 503-022 & $22+4$ & $\cdots$ \\
\hline ESO 504-010 & $22-14$ & 17 \\
\hline ESO 440-039 & $20-5+4$ & 21 \\
\hline ESO 440-049 & $22-5+4$ & 21 \\
\hline ESO 508-034 & $11-22$ & 16 \\
\hline ESO 445-007 & $11-0+22$ & 15 \\
\hline ESO 510-026 & $11-0$ & 28.5 \\
\hline ESO 318-024 & $54+4$ & 9 \\
\hline ESO 440-004 & $22-4$ & 17 \\
\hline
\end{tabular}


Table 1 -Continued

\begin{tabular}{clc}
\hline \hline Galaxy & \multicolumn{1}{c}{ Group } & $\mathrm{D}(\mathrm{Mpc})$ \\
\hline ESO 504-025 & $22-4$ & 17 \\
ESO 507-065 & $11-23+22^{*}$ & $\ldots$ \\
ESO 443-083 & $11+32^{*}$ & $\cdots$ \\
& & \\
\hline
\end{tabular}

*Denotes group for which Tully et al. (1992) do not provide a distance estimate.

Note. - The first column is galaxy name from the combined ELT sample; the second column give corresponding assignment of the galaxy to the galaxy groups defined by Tully (1988). Galaxies within a $6^{\circ}$ projected radius around the center of the Fornax cluster were assumed to be members of Fornax (Bureau et al. 1996). The last column gives the revised distance assigned to the galaxy based on the group assignment. These distances are used in Fig. 5. 


\section{REFERENCES}

Aaronson, M., Huchra, J. P., \& Mould, J. R. 1979, ApJ, 229, 1

Allen, C. W. 1963, Astrophysical Quantities (The Athlone Press, London)

Babu, G. J. \& Feigelson, E. D. 1996, Astrostatistics (Chapman \& Hall, London), p.119-128.

Bergvall, N. \& Rönnback, J. 1995, MNRAS, 273, 603

Bershady, M. A. 1997, in Dark and Visible Matter in Galaxies, edited by M. Persic and P. Salucci (ASP, San Francisco), Vol. 117, p. 537

Bica, E., Arimoto, N. \& Alloin, D. 1988, A\&A, 202, 8

Bottinelli, L., Gouguenheim, L., Paturel, G., \& de Vaucouleurs, G. 1983, A\&A, 118, 4

Bureau, M., Mould, J. R., \& Staveley-Smith, L. 1996, ApJ, 463, 60

Calzetti, D., Kinney, A. L., \& Storchi-Bergmann, T. 1994, ApJ, 429, 582

Carignan, C. \& Beaulieu, S. 1989, ApJ, 347, 760

Côté, S., Carignan, C., \& Sancisi, R. 1991, AJ, 102, 904

Côté, S., Freeman, K., \& Carignan, C. 1997, in Dark and Visible Matter in Galaxies, edited by M. Persic and P. Salucci (ASP, San Francisco), Vol. 117, p. 52

Dalcanton, J. J., Spergel, D. N., \& Summers, F. J. 1997, ApJ, 482, 659

de Blok, W. J. G., McGaugh, S. S., \& van der Hulst, J. M. 1996, MNRAS, 283, 18

de Blok, W. J. G., van der Hulst, J. M., \& Bothun, G. D. 1995, MNRAS, 274, 235

de Vaucouleurs, G., de Vaucouleurs, A., \& Corwin, H. G., Jr. 1976, Second Reference Catalogue of Bright Galaxies (University of Texas Press, Austin)

de Vaucouleurs, G., de Vaucouleurs, A., Corwin, H. G., Buta, R. J., Paturel, G., \& Fouqué, P. 1991, The Third Reference Catalogue of Bright Galaxies (Springer-Verlag, New York) (RC3)

Dickey, J. M., Hanson, M. M., \& Helou, G. 1990, ApJ, 352, 522

Fioc, M. \& Rocca-Volmerange, B. 1997 A\&A, 326, 950

Fouqué, P., Bottinelli, L., Durand, N., Gouguenheim, L., \& Paturel, G. 1990, A\&AS, 86, 473

Franx, M. \& de Zeeuw, T. 1992, ApJ, 392, L47

Freedman, W. L., Madore, B. F., \& Kennicutt, R. C. 1997, in the Extragalactic Distance Scale, edited by M. Levio, M. Donahue, \& N. Panagia, (Cambridge University Press, Cambridge)

Freeman, K. L. 1997, Publ. Astron. Soc. Aust., 14, 4

Gallagher, J. S. III, Littleton, J. E., \& Matthews, L. D. 1995, AJ, 109, 2003

Han, M. 1992, ApJ, 391, 617

Heyl, J. S., Cole, S., Frenk, C., \& Navarro, J. 1995, MNRAS, 274, 755

Hunter, D. A. \& Gallagher, J. S. 1986, PASP, 98, 5

Jobin, M. \& Carignan, C. 1990, AJ, 100, 648

Kamphuis, J. \& Sancisi, R. 1993, A\&A, 273, L31

Karachentsev, I. D. 1991, Sov. Astron. Let., 17, 283

Kormendy, J. 1990, in Evolution of the Universe of Galaxies, ASP Conference Series, Vol. 10, edited by R. G. Kron, (Astronomical Society of the Pacific, San Francisco), p. 33 
Lake, G., Schommer, R. A., \& van Gorkom, J. H. 1990, AJ, 99, 547

Lauberts, A. \& Valentijn, E. A. 1989, The Surface Photometry Catalogue of the ESO-Uppsala Galaxies (European Southern Observatory, Garching bei München)

Levine, S. E. \& Sparke, L. S. 1998, ApJ, 496, 13

Matthews, L. D. \& Gallagher, J. S. III. 1997, AJ, 114, 1899 (MG)

Matthews, L. D. \& Gallagher, J. S. III. 1998, in preparation

Matthews, L. D., Gallagher, J.S. III, \& van Driel, W. 1997, in Dark and Visible Matter in Galaxies, edited by M. Persic and P. Salucci (ASP, San Francisco), Vol. 117, p. 98

Matthews, L. D., van Driel, W., \& Gallagher, J.S. III. 1998, AJ, in press

McGaugh, S. S. 1994, ApJ, 426, 135

McGaugh, S. S. \& Bothun, G. D. 1994, AJ, 107, 530

Meurer, G. R., Carignan, C., Beaulieu, S. F., \& Freeman, K. C. 1996, AJ, 111, 1551

Meurer, G. R., Mackie, G., \& Carignan, C. 1994, AJ, 107, 2021

Milgrom, M. \& Braun, E. 1988, ApJ, 334, 130

Mo, H. J., Mao, S., \& White, S. D. M. 1998, MNRAS, 295, 319

Persic, M. \& Salucci, P. 1991, MNRAS, 248, 325

Pierce, M. J. 1998, private communication

Pierce, M. J. \& Tully, R. B. 1988, ApJ, 330, 579

Pierce, M. J. \& Tully, R. B. 1992, ApJ, 387, 47 (PT)

Pisano, D. J., Wilcots, E. M., \& Elmegreen, B. G. 1998, AJ, 115, 975

Rhee, M.-H. 1996, Ph.D. Thesis, University of Groningen

Roberts, M. S., 1978, AJ, 83, 1026

Romanishin, W., Krumm, N., Salpeter, E., Knapp, G., Strom, K. M., \& Strom, S. E. 1982, ApJ, 263, 94

Rönnback, J. \& Bergvall, N. 1994, A\&AS, 108, 193

Rönnback, J. \& Bergvall, N. 1995, A\&A, 302, 353

Rownd, B. K., Dickey, J. M., \& Helou, G. 1994, AJ, 108, 1638

Salpeter, E. E. \& Hoffman, G. L. 1996, ApJ, 465, 595

Salucci, P. \& Persic, M. 1997, in Dark and Visible Matter in Galaxies, edited by M. Persic and P. Salucci, (ASP, San Francisco), Vol. 117, p.1

Sandage, A. \& Tammann, G. 1981, A Revised Shapely-Ames Catalog of Bright Galaxies (Carnegie Institution, Washington)

Shaya, E. J., Tully, R. B., \& Pierce, M. J. 1992, ApJ, 391, 16

Silbermann, N. A, et al. 1998, to appear in ApJ

Skillman, E. D. 1996 in The Minnesota Lectures on Extragalactic Neutral Hydrogen, ASP Conference Series, Vol. 106, edited by E. D. Skillman (ASP, San Francisco), p.208

Skillman, E. D., Bothun, G. D., Murray, M. A., \& Warmels, R. H. 1987, A\&A, 185, 61

Somerville, R. S. \& Primack, J. R. 1998, MNRAS, submitted

Sprayberry, D., Bernstein, G. M., Impey, C. D., \& Bothun, G. D. 1995, ApJ, 438, 72 
Tully, R. B. 1988, Nearby Galaxies Catalog (Cambridge University Press, Cambridge) (TNGC)

Tully, R. B. \& Fisher, J. R. 1977, A\&A, 54, 661

Tully, R. B. \& Fisher, J. R. 1987, Nearby Galaxies Atlas, (Cambridge University Press, Cambridge)

Tully, R. B. \& Fouqué, P. 1985, ApJS, 58, 67 (TFq)

Tully, R. B., Pierce, M. J., Huang, J.-S., Saunders, W., Verheijen, M. A. W., \& Witchalls, P. 1998, AJ, 115, 2264

Tully, R. B., Shaya, E. J., \& Pierce, M. J. 1992, ApJS, 80, 479

Tully, R. B., Verheijen, M. A. W., Pierce, M. J., Huang, J.-S., \& Wainscoat, R. J. 1996, AJ, 122, 2471

Vader, J. P. \& Chaboyer, B. 1994, AJ, 108, 1209

Vennik, J., Richter, G. M., Thänert, W., \& Biering, C. 1996, Astron. Nachr., 317, 289

Walsh, W. M. 1998, private communication

White, S. \& Frenk, C. 1991, ApJ, 379, 52

Young, L. M. \& Lo, K. Y. 1996, ApJ, 462, 203

Zwaan, M. A., van der Hulst, J. M., de Blok, W. J. G., \& McGaugh, S. S. 1995, MNRAS, 273, L35 


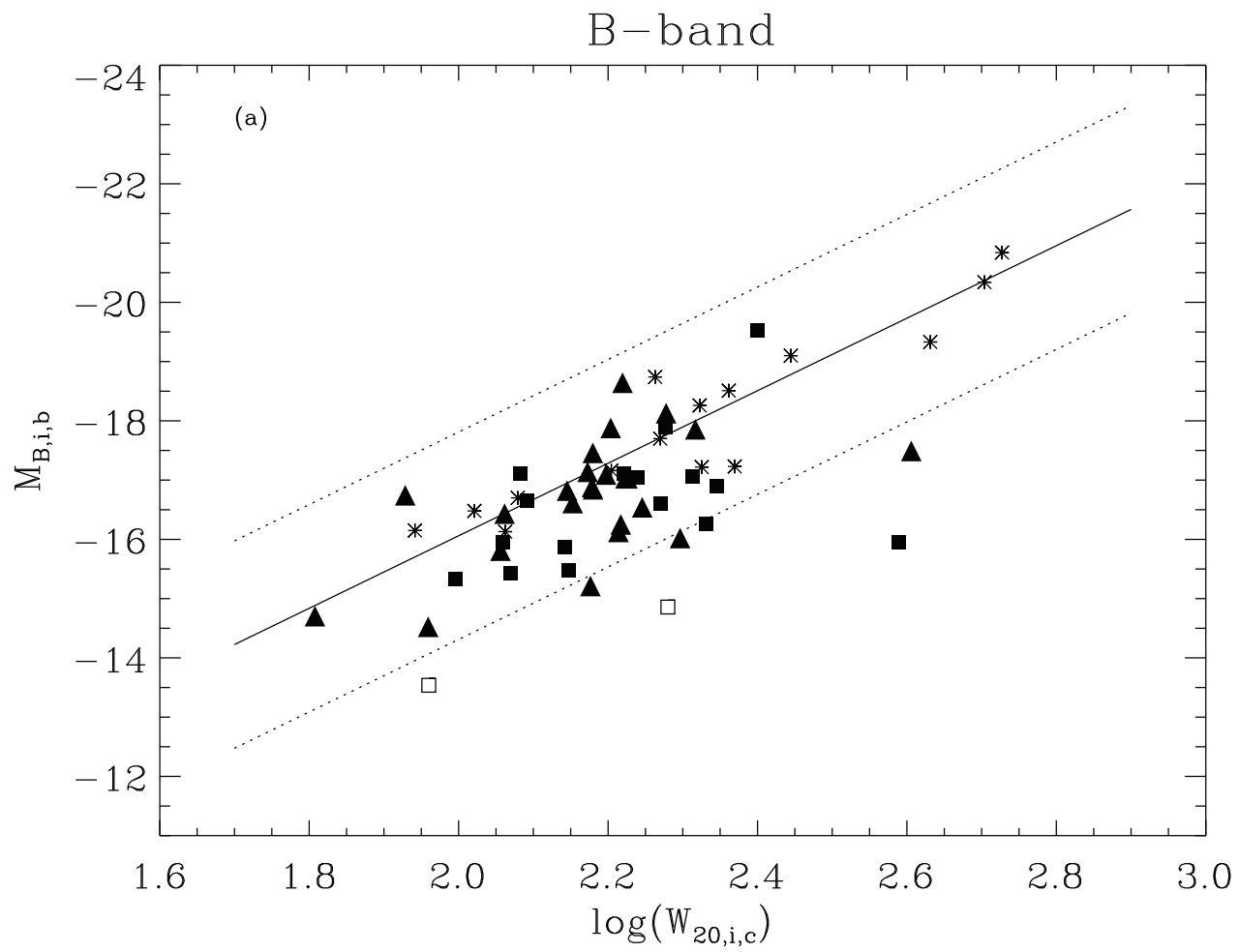

Fig. 1a.- $B$-band Tully-Fisher relation for the combined ELT sample and the local calibrator sample of PT. Triangles are galaxies from Paper I; filled squares are 17 additional extreme late-type spirals from MG; asterisks are the PT sample. Distances to the combined ELT sample galaxies are derived using a simple linear Hubble flow with $H_{o}=85 \mathrm{~km} \mathrm{~s}^{-1} \mathrm{Mpc}^{-1}$. The axes are the logarithm of the observed $\mathrm{H}$ I linewidth at $20 \%$ peak maximum, in $\mathrm{km} \mathrm{s}^{-1}$, corrected for turbulence and inclination, versus the $B$-band absolute magnitudes, corrected for Galactic and internal extinction, as described in the Text. In Panel $(a)$, the open squares represent the galaxies DDO 154 (on the left) and NGC 2915 (on the right; see Sect. 2.5.1). The solid line represents a least squares fit to the PT data, and the dotted lines indicate the $2 \sigma$ scatter. 


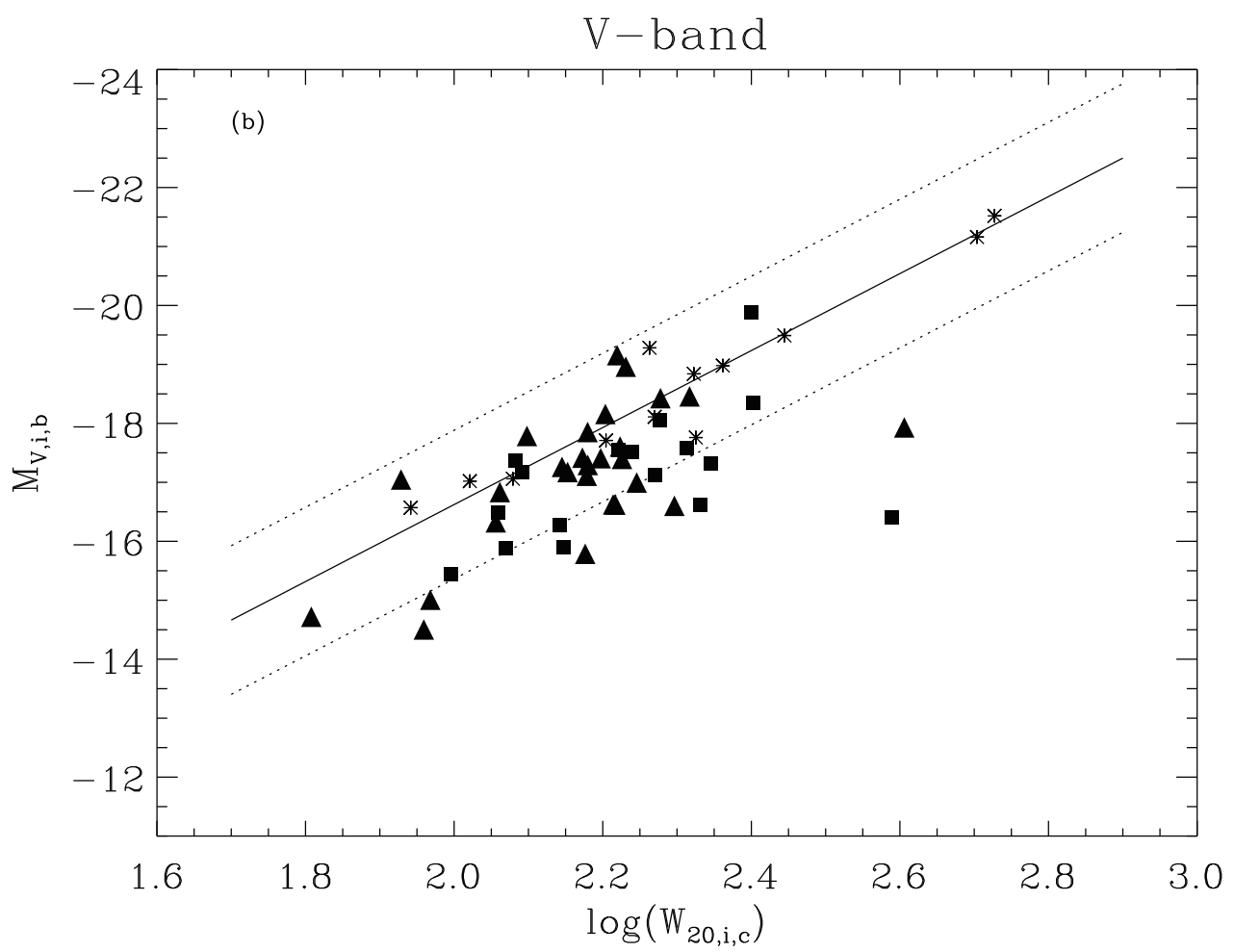

Fig. 1b. - $V$-band Tully-Fisher relations for the combined ELT sample and the local calibrator sample of PT. See Figure 1a for details. 


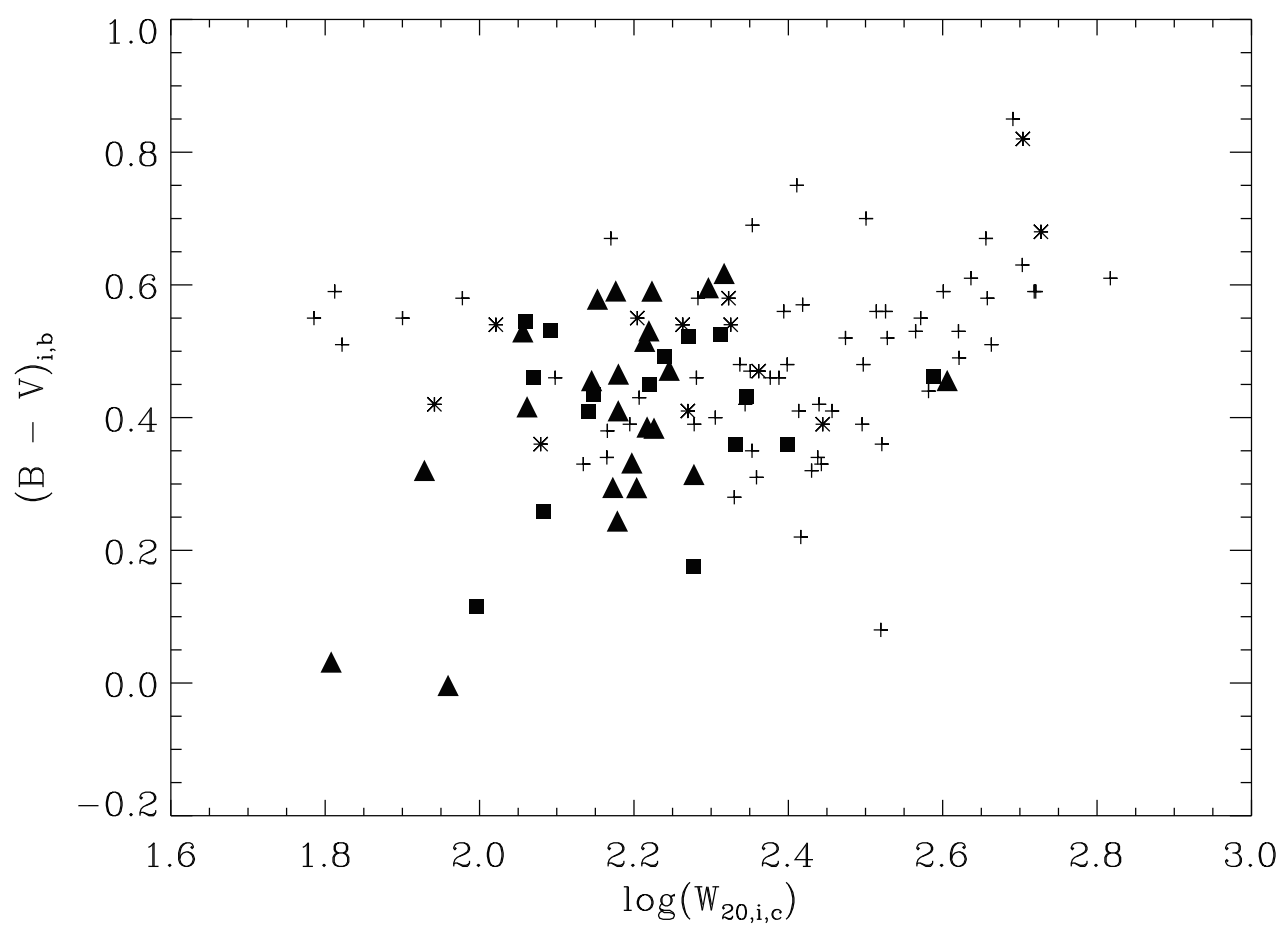

Fig. 2.- The logarithm of the observed H I linewidth at $20 \%$ peak maximum, in $\mathrm{km} \mathrm{s}^{-1}$, corrected for turbulence and inclination, versus $B-V$ color, corrected for internal and Galactic extinction. Pluses are galaxies from the TNGC; the remaining symbols are as in Fig. 1. 


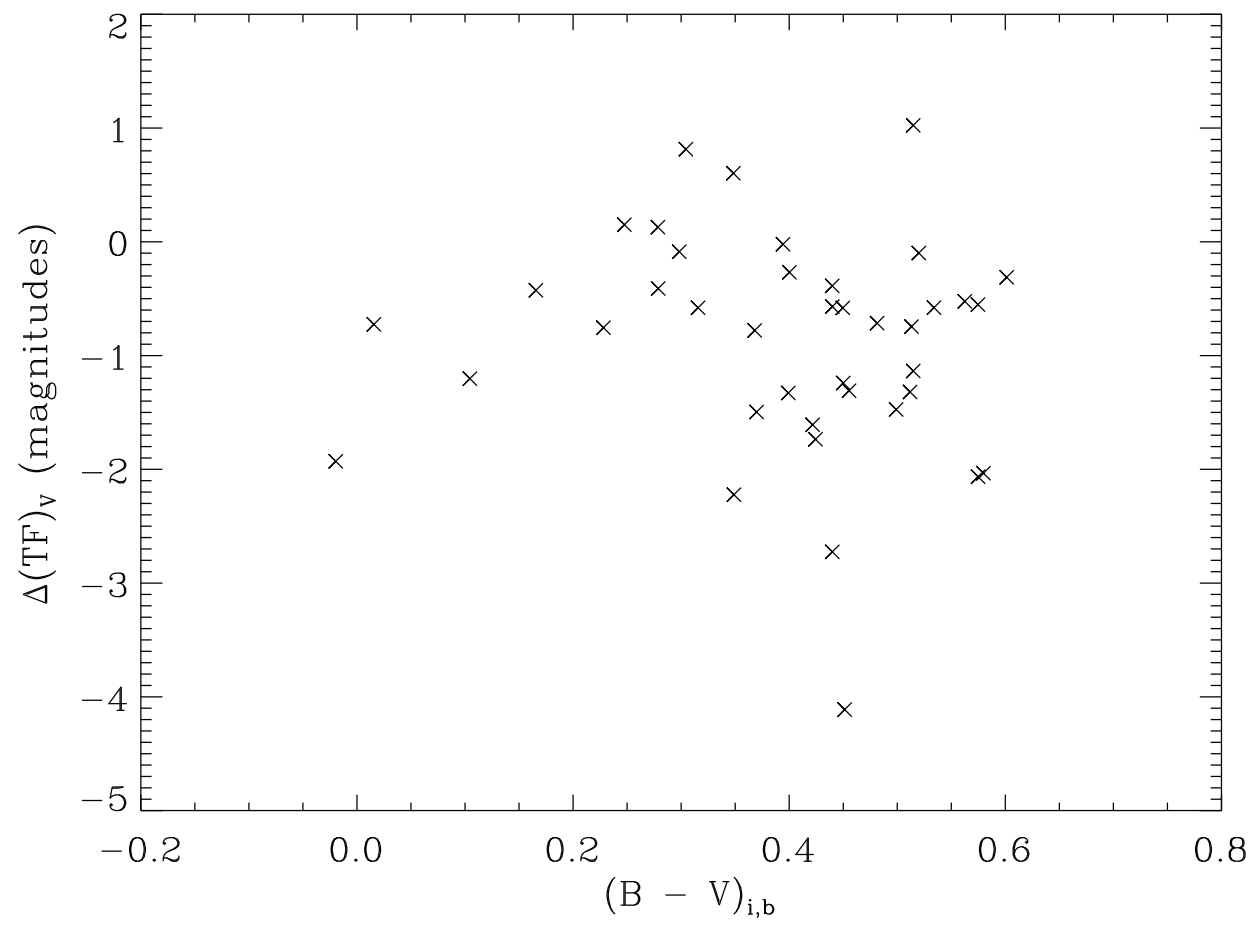

Fig. 3.- Deviation of the combined ELT sample from the standard $V$-band Tully-Fisher relation, in magnitudes, versus $B-V$ color, corrected for Galactic and internal extinction. 


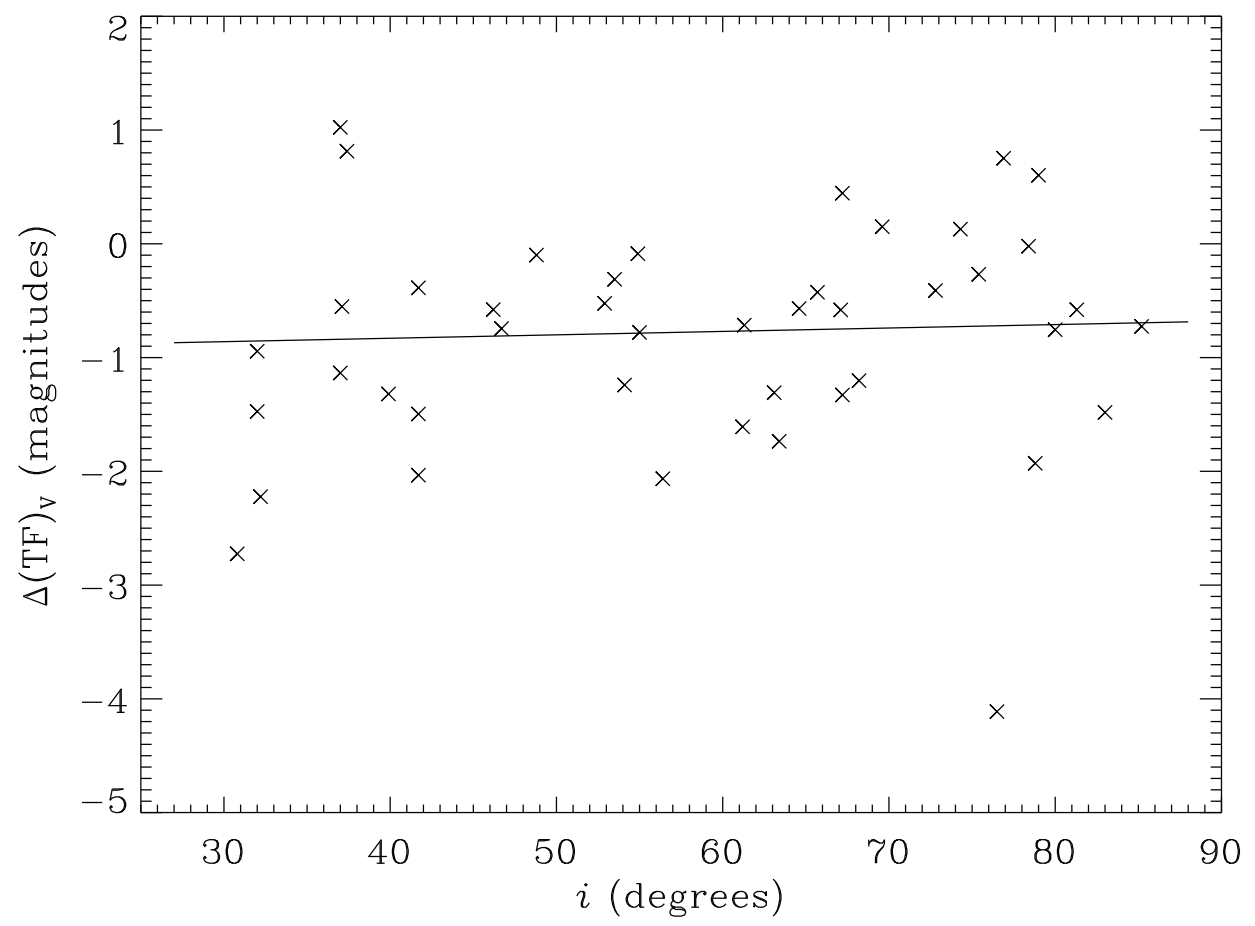

Fig. 4.- Deviation of the combined ELT sample from the standard $V$-band Tully-Fisher relation, in magnitudes, versus inclination, in degrees. The solid line shows a least squares fit to the data. 


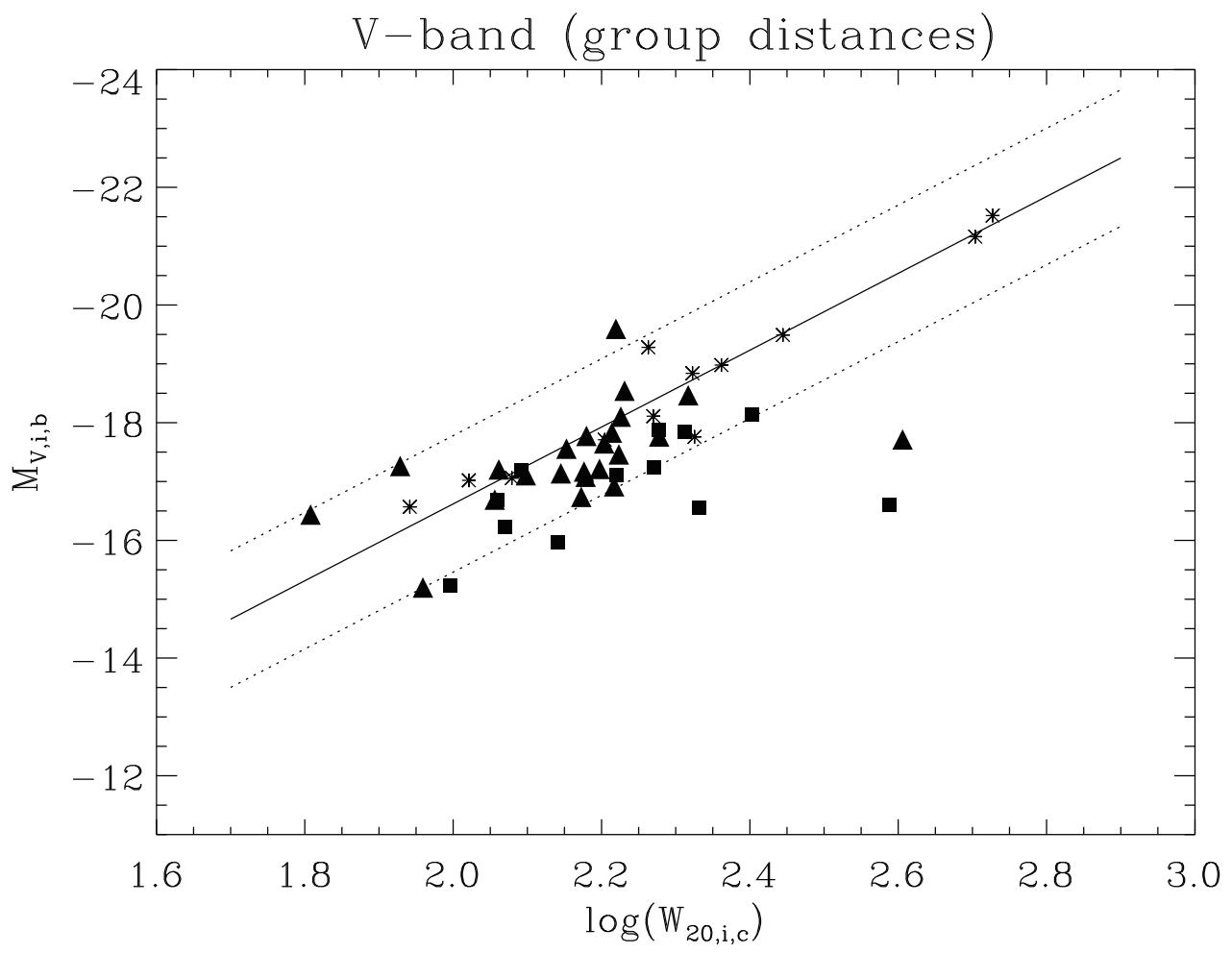

Fig. 5.- Same as Fig. 1 (a), except that distances to the combined ELT sample galaxies have been derived using galaxy group assignments (see Sect. 2.4.5). 


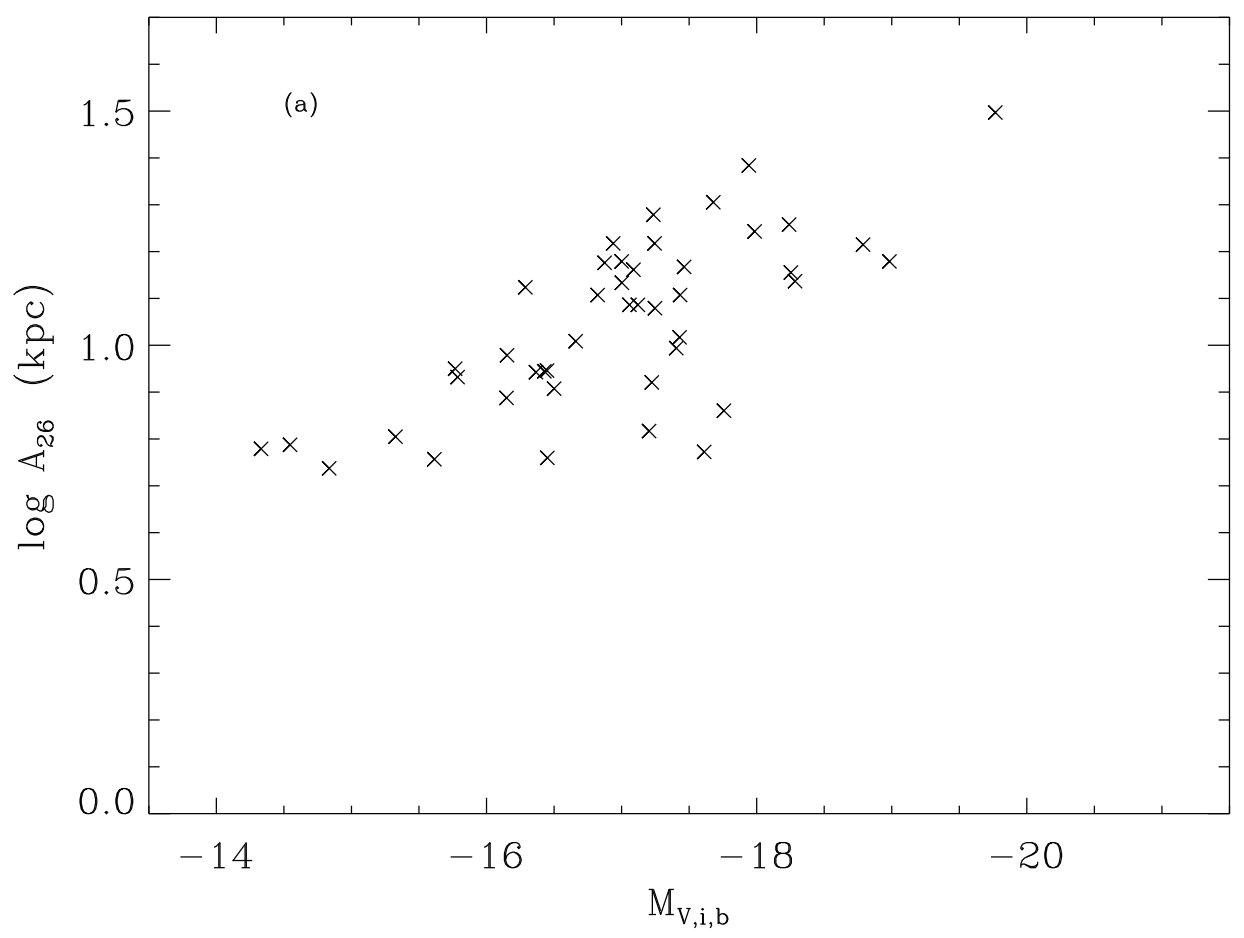

Fig. 6a.- The relationship between absolute $V$ magnitude, corrected for internal and external extinction, and the logarithm of the optical linear size (in kiloparsecs) at the 26 magnitude per arcsecond squared isophote for the combined ELT sample galaxies. Panel $(a)$ assumes distances derived from a linear Hubble law. 


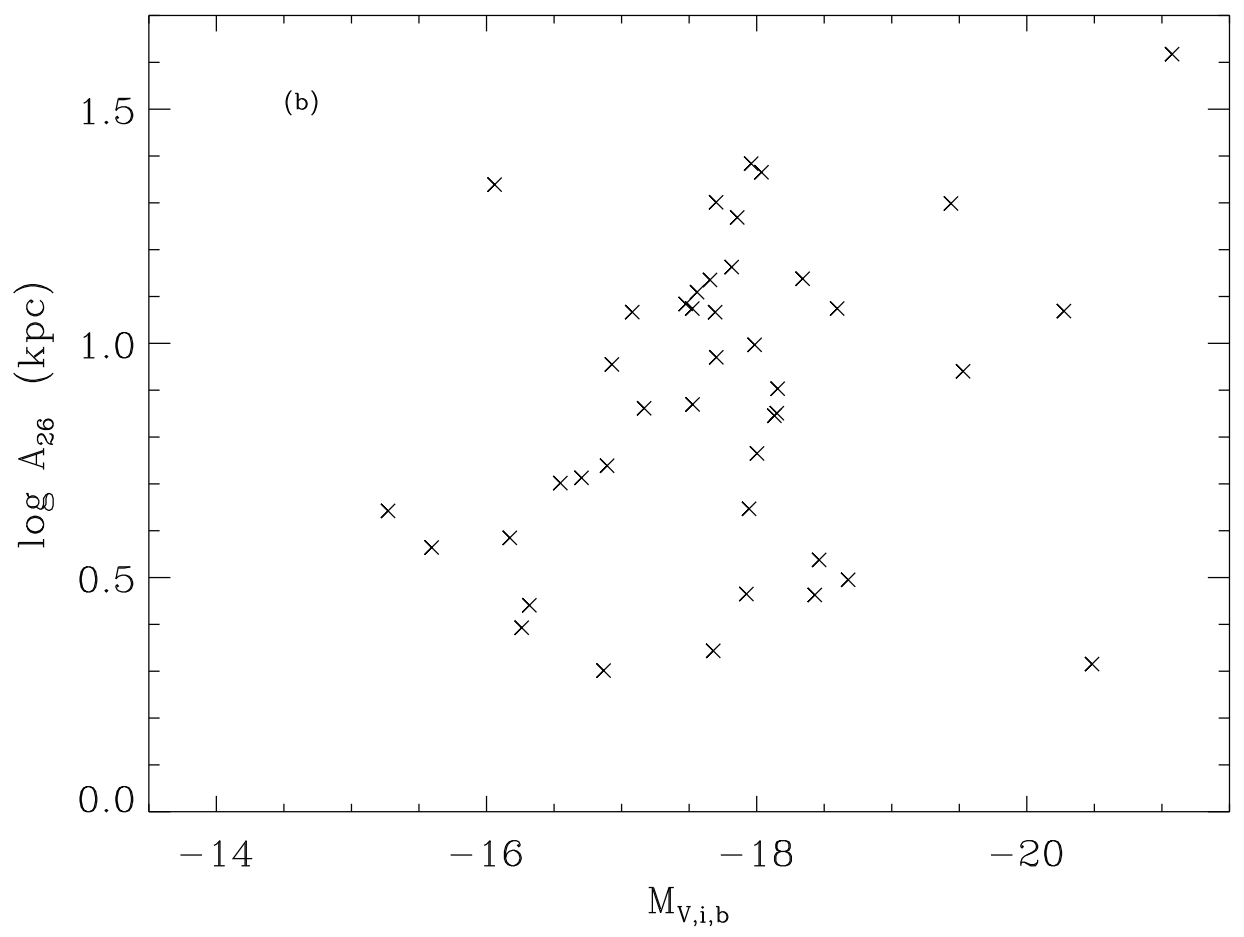

Fig. 6b. - Same as Figure 6a but panel (b) shows the resulting relation if distances to the galaxies are derived by assuming the galaxies adhere to the standard $V$-band TF relation shown in Fig. $1(b)$. 


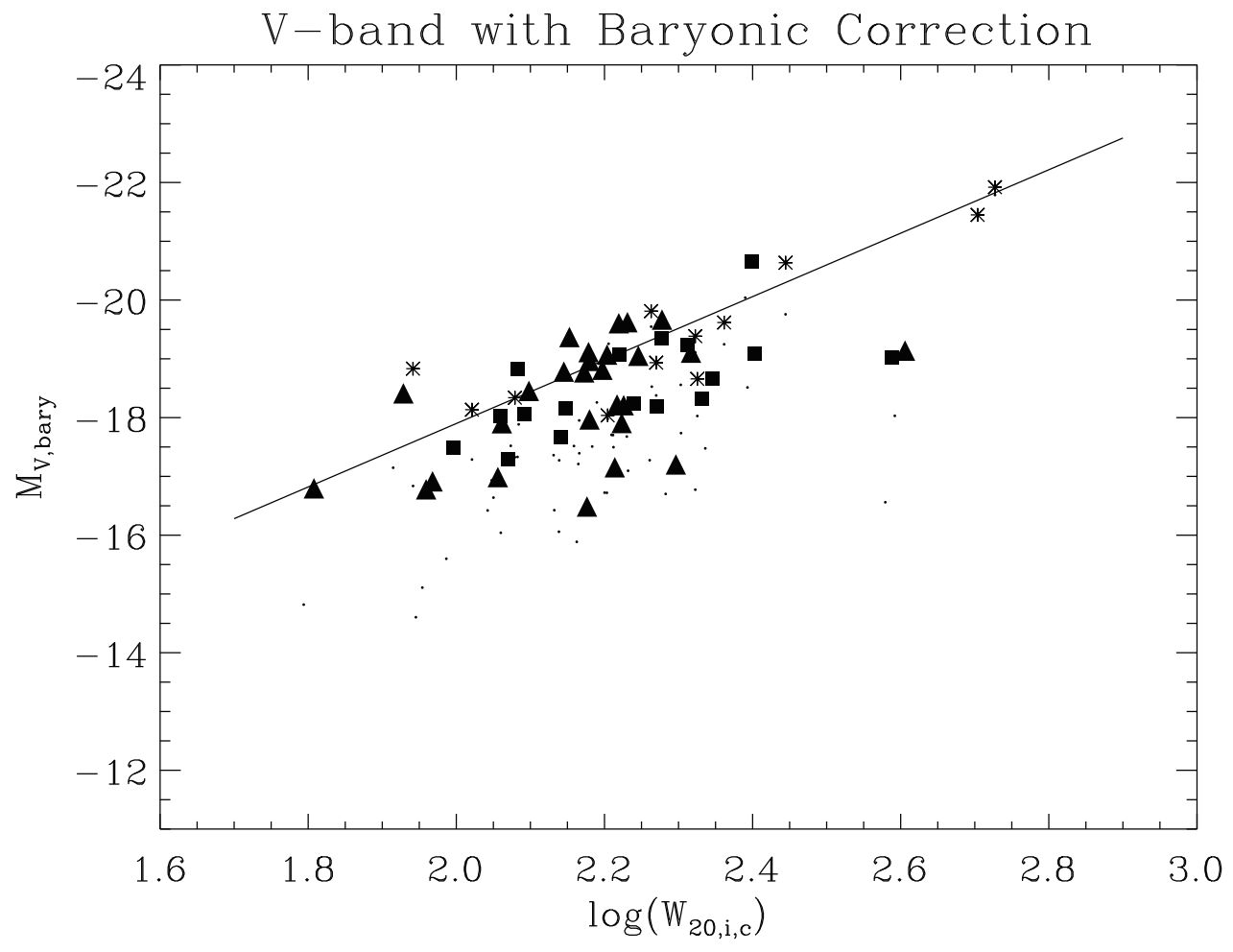

Fig. 7.- Same as Fig. 1 (b), except that baryonic corrections (see Sect. 2.5) have been applied to the luminosities of both the PT and the combined ELT sample galaxies. Small dots indicate positions of galaxies before the baryonic correction. The solid line is a least squares fit to the PT data. Distances to the combined ELT sample galaxies are derived assuming a linear Hubble law. 


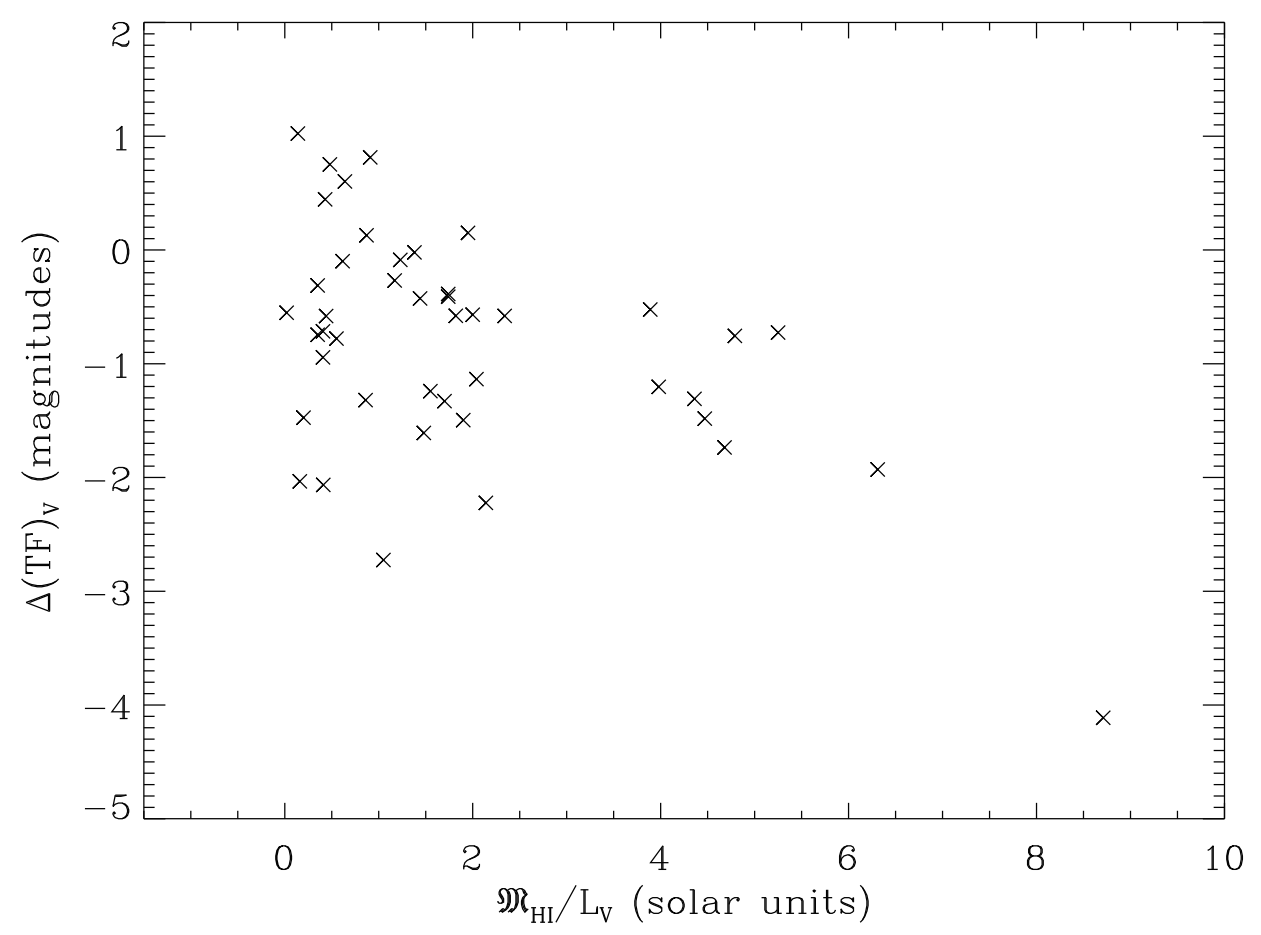

Fig. 8.- Deviation of the combined ELT sample from the standard $V$-band Tully-Fisher relation, in magnitudes (assuming distances derived from a linear Hubble flow), versus the ratio of the neutral hydrogen mass to the optical $V$-band luminosity, in solar units. 


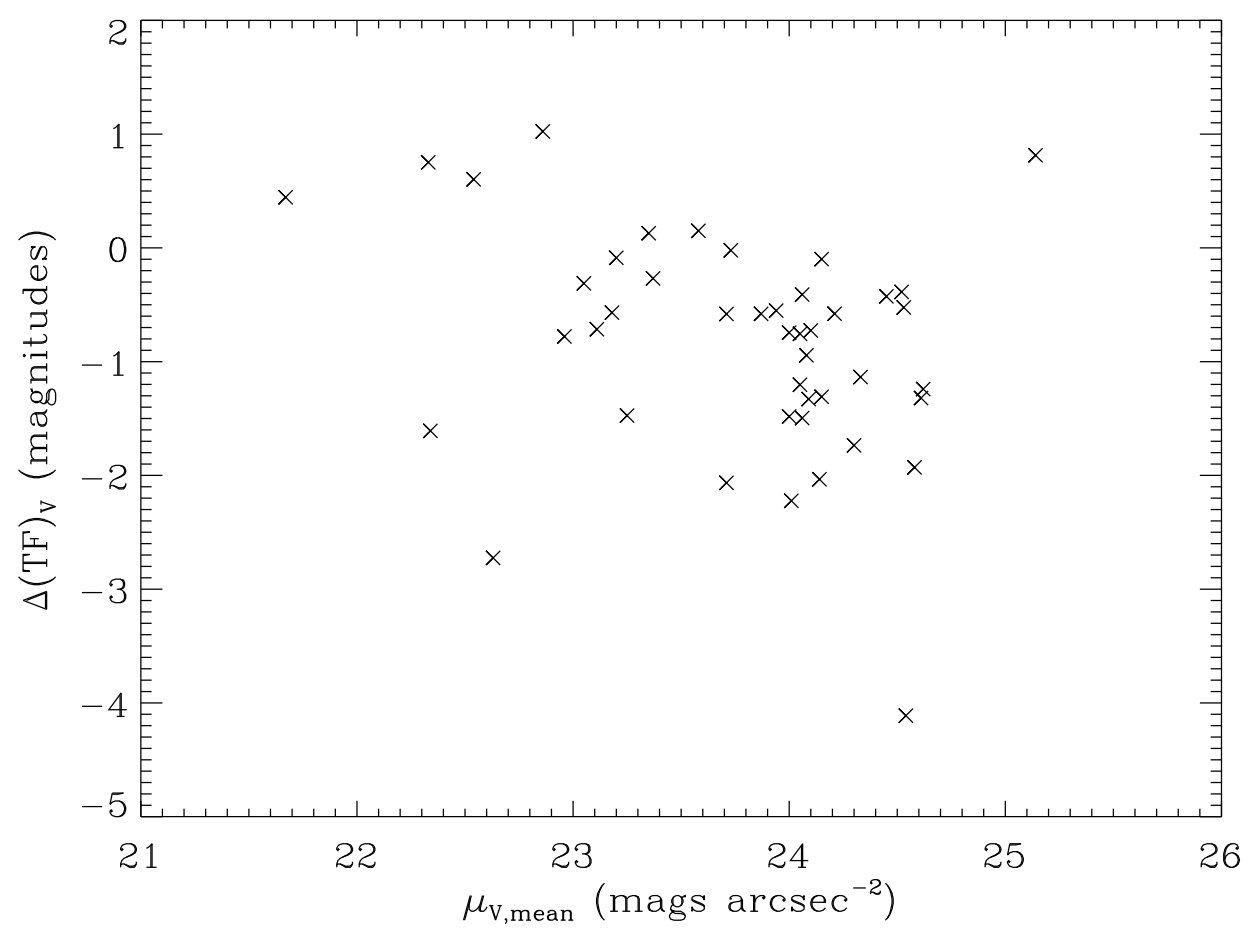

Fig. 9.- Deviation of the combined ELT sample from the standard $V$-band Tully-Fisher relation, in magnitudes (assuming distances derived from a linear Hubble flow), versus the mean, observed $V$ band disk surface brightness, in magnitudes per arcsecond squared. Mean disk surface brightnesses are taken from MG. 


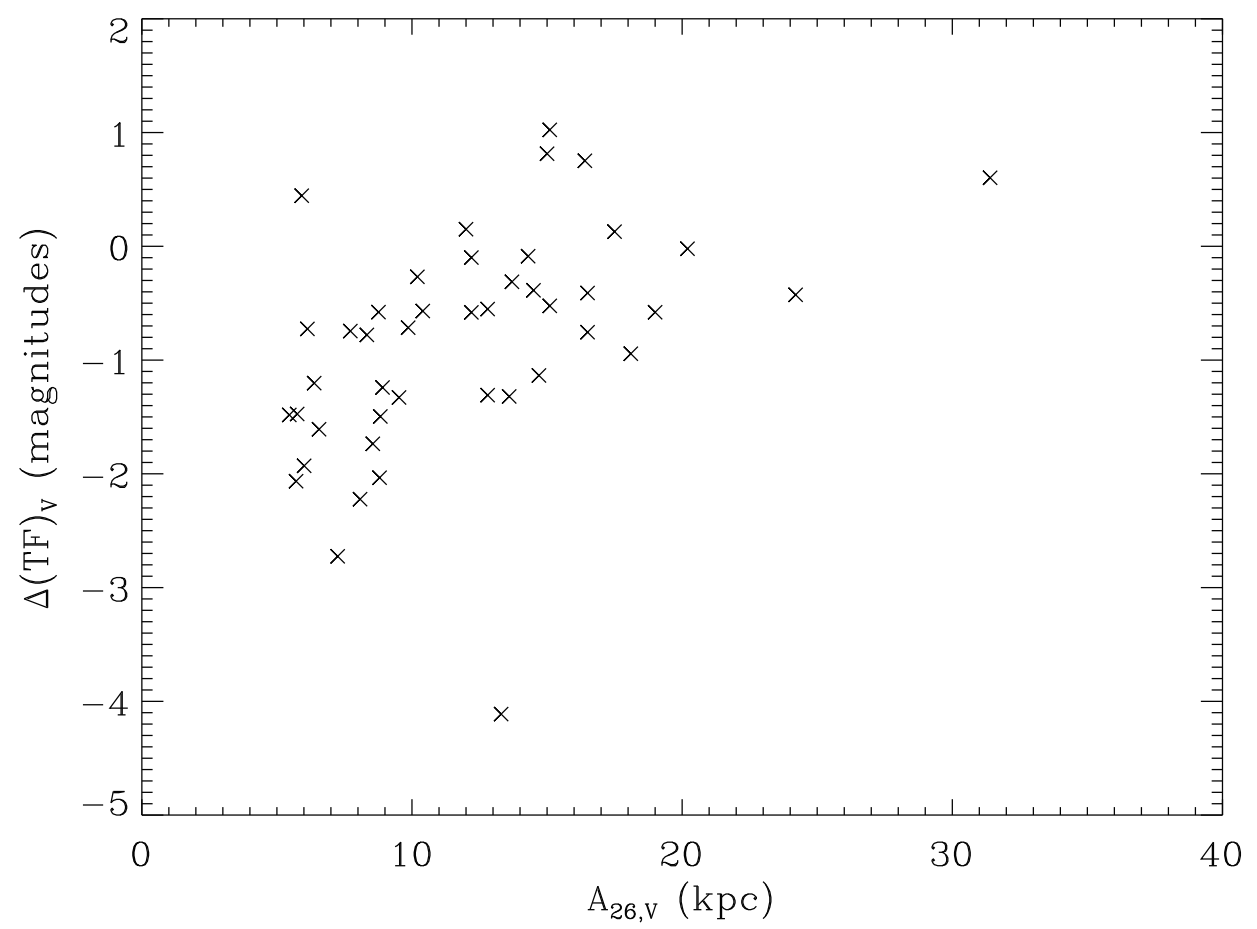

Fig. 10.- Deviation of the combined ELT sample from the standard $V$-band Tully-Fisher relation, in magnitudes (assuming distances derived from a linear Hubble flow), versus the $V$-band optical linear diameter (in kiloparsecs) at the 26 magnitude per arcsecond squared isophote. Distances using a linear Hubble law are assumed for the combined ELT sample galaxies. The optical linear sizes are derived using the angular diameters of MG. 


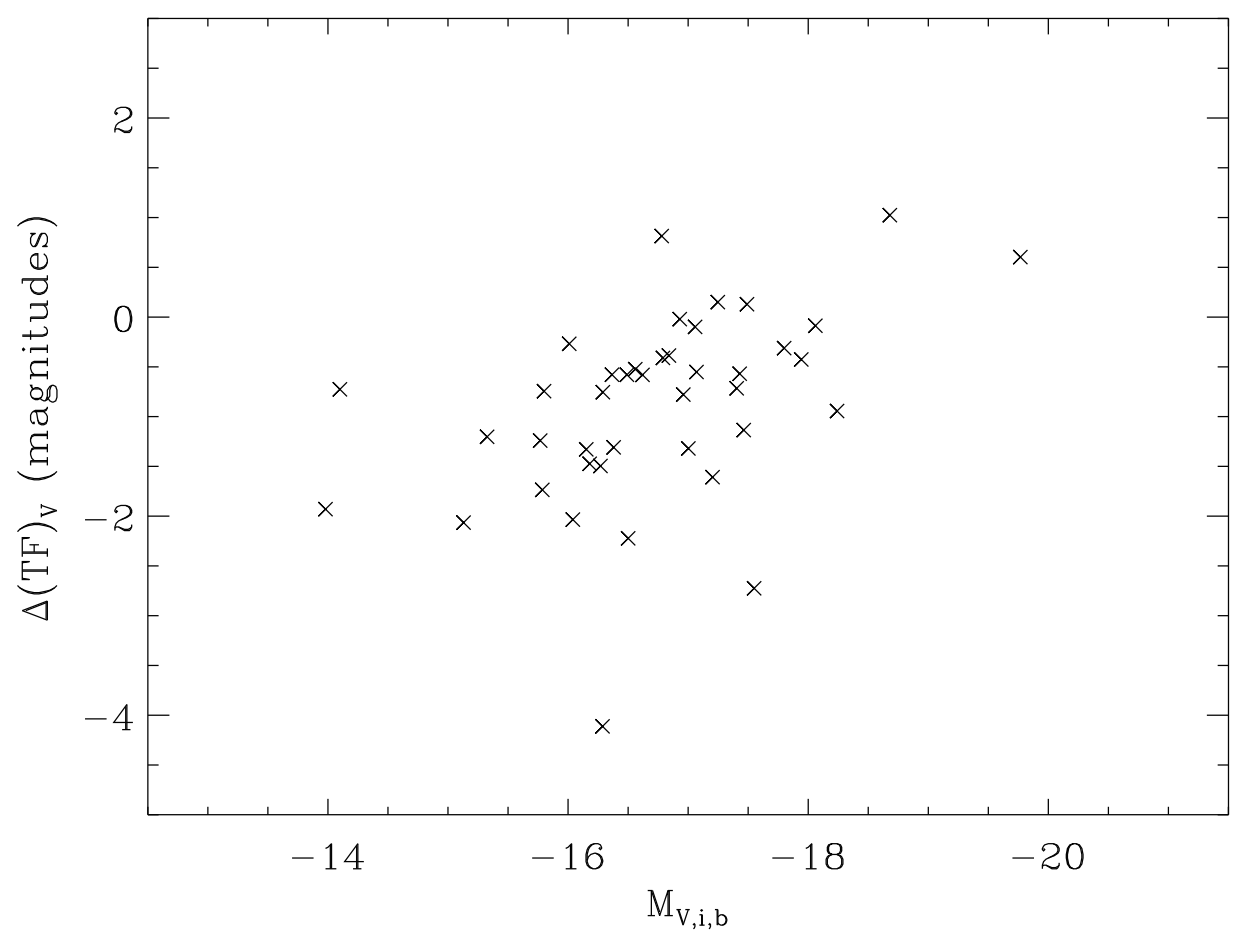

Fig. 11. - Deviation of the combined ELT sample from the standard $V$-band Tully-Fisher relation, in magnitudes (assuming distances derived from a linear Hubble flow), versus the absolute $V$-band magnitude, corrected for internal and external extinction. 